\title{
Melanoma cutâneo: estudo prospectivo de 42 casos $^{*}$
}

\author{
Cutaneous melanoma: a prospective study of 42 cases
}

\author{
Nurimar Conceição Fernandes ${ }^{1}$
}

Roberto Calmon ${ }^{2}$

\begin{abstract}
Resumo: Estudo longitudinal prospectivo de 42 casos de melanoma cutâneo revelou: $71,2 \%$ entre 50 e 79 anos; distribuição etária homogênea entre os gêneros masculino $(45,1 \%)$ e feminino $(54,7 \%)$; predominância de brancos (88\%); localização no tronco $(54,7 \%)(\mathrm{p}=0,039)$; tipo clínico-histológico expansivo superficial $(52,3 \% / 26,1 \%)(p=0,02) ; 16$ casos $(38,1 \%)$ IA e nove melanomas in situ $(21,4 \%)$.

Palavras-chave: Diagnóstico; Epidemiologia; Melanoma; Neoplasias cutâneas; Patologia

Abstract: A prospective longitudinal study of 42 cases of cutaneous melanoma showed that: $71.2 \%$ of patients were between 50 and 79 years of age; $45.1 \%$ were male and $54.7 \%$ female; most patients (88\%) were white; most lesions $(54.7 \%)$ were located on the trunk $(\mathrm{p}=0.039)$; the most common clinical and histological pattern was the superficial spreading melanoma $(52.3 \% / 26.1 \%)(p=0.02) ; 16$ cases $(38.1 \%)$ were classified as stage IA and 9 cases (21.4\%) consisted of melanomas in situ.

Keywords: Diagnosis; Epidemiology; Melanoma; Pathology; Skin neoplasms
\end{abstract}

São fatores de risco para o melanoma cutâneo a exposição solar, o fenótipo do paciente, a história familiar e melanoma prévio. O estádio do tumor no momento do diagnóstico é o principal fator prognóstico. Cirurgia é o tratamento-padrão inicial para doença localizada. A biópsia do linfonodo sentinela é a abordagem indicada para o estadiamento nodal. Em pacientes metastáticos, a bioterapia ou quimioterapia sistêmica pode ser tentada, enquanto a radioterapia deve ser considerada paliativa.

No período de 2004-2008, em estudo longitudinal prospectivo, 42 casos de melanoma cutâneo no Hospital Universitário Clementino Fraga Filho, Universidade Federal do Rio de Janeiro, foram submetidos a:

a) biópsia excisional com margem de $1 \mathrm{~cm}$ (na face ou em caso de linfonodo sentinela); o índice de Breslow definiu as margens ótimas: < $1 \mathrm{~mm}$ $(1 \mathrm{~cm})$ e $>1 \mathrm{~mm}(2 \mathrm{~cm})$, e a necessidade de ampliação de margens; $;^{1,2}$

b) biópsia incisional (punch $6 \mathrm{~mm}$ ) na face, no pavilhão auricular, nas regiões palmoplantares $\mathrm{e}$ subungueais; ${ }^{1,2}$ c) estadiamento clínico: palpação dos linfonodos regionais; se assimétricos, duros ou pétreos, ultrassonografia (USG) para avaliação de metástase (aspecto globoso, hipoecogenicidade; superfície bosselada com/sem alterações hilares; aumento de volume); USG com Doppler para avaliação de angioarquitetura. ${ }^{3,4}$ Em seguida, punção nodal com agulha fina para citologia do aspirado e/ou biópsia. ${ }^{5,6}$ Dissecção ganglionar terapêutica quando confirmado o comprometimento nodal;

d) hemograma, velocidade de hemossedimentação (VHS), desidrogenase lática (LDH), hepatograma, radiografia (RX) de tórax (na ausência de sinais e sintomas);

e) TC (crânio, tórax, abdominopélvica), cintilografia óssea, RX de esqueleto (nos casos com sintomas à distância);

f) abordagem cirúrgica foi realizada no setor de Cirurgia Dermatológica e Serviços de Cirurgia Plástica (ressecção de lesões), Cirurgia-Geral (punção / biópsia de linfonodo / esvaziamento ganglionar) e Ortopedia (amputações).

g) análise estatística pelo teste quiquadrado.

Aprovado pelo Conselho Consultivo e aceito para publicação em 27.12.10.

* Trabalho realizado nos Serviços de Dermatologia e Anatomia Patológica e Oncologia Clínica do Hospital Universitário Clementino Fraga Filho - Universidade Federal do Rio de Janeiro (HUCFF - UFRJ) - Rio de Janeiro (RJ), Brasil.

Conflito de interesse: Nenhum / Conflict of interest: None

Suporte financeiro: Nenhum / Financial funding: None

Doutor; professor-associado da Universidade Federal do Rio de Janeiro (UFRJ) - Rio de Janeiro (RJ), Brasil

Doutor; médico do Serviço de Oncologia Clínica do Hospital Universitário Clementino Fraga Filho - Universidade Federal do Rio de Janeiro (HUCFF - UFRJ) - Rio de Janeiro (RJ), Brasil 
TABela 1: Distribuição dos casos segundo faixa etária, gênero e cor

\begin{tabular}{lllllll}
\hline & \multicolumn{2}{c}{ Brancos } & & \multicolumn{2}{c}{ Não brancos } & \\
\cline { 2 - 3 } Faixa etária & Masculino & Feminino & & Masculino & Feminino & Total (\%) \\
\hline $20-29$ & 1 & - & - & - & $1(25)$ \\
$30-39$ & 1 & 1 & - & 1 & $3(7)$ \\
$40-49$ & 1 & 2 & & - & 1 & $7(7)$ \\
$50-59$ & 5 & 1 & & & 1 & $7(16,6)$ \\
$60-69$ & 4 & 2 & & - & 1 & $16(38)$ \\
$70-79$ & 5 & 9 & & 1 & - & $5(11,9)$ \\
$80-89$ & 1 & 4 & & $-1(2,3)$ & $4(9,52)$ & $42(100)$ \\
Total (\%) & $18(42,8)$ & $19(45,2)$ & & & \\
\hline
\end{tabular}

Fonte: HUCFF/UFRJ (2004-2008) / p =0,79

A rotina de seguimento deste tratamento é baseada no índice de Breslow, por tempo indeterminado, exceto para melanomas in situ, que permitem alta dos pacientes. ${ }^{1} \mathrm{O}$ American Joint Committee on Cancer (AJCC 2002/2009) não define rotinas especiais. ${ }^{78}$ A técnica do linfonodo sentinela atendeu as recomendações do AJCC 2002/2009 (linfonodos regionais clinicamente negativos, Breslow $1 \mathrm{~mm}$ e $4 \mathrm{~mm}$ ) e às do GBM (Breslow $330,76 \mathrm{~mm}$; <0,76mm associado a ulceração e/ou regressão histológica).

A distribuição etária entre os gêneros foi homogênea, predominando brancos e a faixa de 70-79 anos $(38 \%)(p=0,79)$ (Tabela 1). O melanoma expansivo superficial (MES) ocorreu em 22 casos (52,3\%), sendo 15 mulheres $(\mathrm{p}=0,022)$ (Tabela 2$)$. A localização no tronco ocorreu em 23 casos $(54,7 \%) \quad(p=0,039)$. Histologicamente, 11 MES (26,1\%), dez melanomas in situ $(23,8 \%)$, sete melanomas lentigo maligno (MLM) (16,6\%), três lentiginosos acrais (MLA) (7,1\%), um desmoplásico $(2,3 \%)$ e dez $(23,8 \%)$ não foram classificados. Segundo estadiamento AJCC 2002 e 2009, houve nove (T0), 16 (IA), dois (IB), cinco (IIA), quatro (IIB), um (IIIA), um (IIIC), três (IV) e um (Tx). ${ }^{1,7,8}$ O caso de melanoma desmoplásico e neurotrópico evoluiu com duas recorrências locais. ${ }^{1}$
Os dois casos de melanoma ungueal submetidos à amputação do hálux evoluíram com doença linfonodal e metástases (pele/pulmão/fígado) no intervalo de um ano. O melanoma subungueal, variante do MLA, surge, em geral, a partir da matriz ungueal no hálux ou polegar. Para o MLA, não há forma de prevenção e seus fatores de risco são totalmente desconhecidos. ${ }^{9,10}$ É um tipo raro: em todos os casos de melanoma na população geral, as incidências relatadas ficaram entre $0,7 \%$ e $3,5 \%$. É também o de pior prognóstico, com a sobrevida em cinco anos, variando de $16-87 \%$.

No caso de melanoma amelanótico - variante rara e de comportamento mais agressivo - apesar do tratamento com interferon, ocorreram metástases dois anos após diagnóstico. Foi observado um caso de MES na região interescapular associado a resíduos de nevo melanocítico intradérmico. $O$ percentual de transformação de nevo melanocítico em melanoma varia de $1-20 \% .{ }^{1}$ O caso de MLA no pé e metástase para quatro linfonodos inguinais recebeu tratamento adjuvante com interferon. Após tratamento, evoluiu com manchas acrômicas na face, que involuíram com hidrocortisona $1 \%$ tópica. Ainda que a co-ocorrência de melanoma metastático e de despigmentação seme-

Tabela 2: Distribuição dos casos segundo cor, gênero e tipo clínico

\begin{tabular}{lllllll}
\hline & \multicolumn{2}{c}{ Brancos } & & \multicolumn{2}{c}{ Não brancos } & \\
\cline { 2 - 3 } Tipo clínico & Masculino & Feminino & & Masculino & Feminino & Total (\%) \\
\hline Expansivo superficial & 7 & 14 & & - & 1 & $22(52,3)$ \\
Lentiginoso acral & 2 & 2 & & 1 & 3 & $8(19)$ \\
Lentigo maligno & 7 & 1 & & - & - & $8(19)$ \\
Nodular & 2 & 2 & & - & - & $4(9,52)$ \\
Total (\%) & 18 & 19 & & 1 & 4 & $42(100)$ \\
\hline
\end{tabular}

Fonte: HUCFF/UFRJ (2004-2008) / p=0,022 
lhante à provocada pelo vitiligo seja conhecida, a relação causal permanece controversa.

Em um paciente, o melanoma se desenvolveu em úlcera de estase tibial esquerda, com satelitose e metástases em trânsito. Ele foi submetido a amputação infrapatelar e esvaziamento ganglionar inguinal (sem metástase). O tecido subcutâneo de uma úlcera está exposto aos agentes ambientais (colonização bacteriana) e a estase venosa pode desencadear hiperplasia epidérmica e proliferação das cristas interpapila- res. Dois pacientes com MLA foram submetidos à linfadenectomia ileoinguinal. Do exame patológico cuidadoso da base linfonodal ressecada inferem-se as seguintes determinantes do estadiamento: número de linfonodos metastáticos; se o tumor é microscópico (clinicamente oculto); se é macroscópico (clinicamente aparente por exame físico e radiológico).

O melanoma cutâneo nesta série mostrou padrão semelhante à anterior (1993-2003), delineando perfil em hospital terciário.

\section{REFERÊNCIAS}

1. Fernandes NC, Calmon R, Maceira JP, Cuzzi T, da Silva CSC. Melanoma cutâneo: estudo prospectivo de 65 casos. An Bras Dermatol. 2005;80:25-34.

2. Lotze MT, Dallal RM, Kirkwood JM, Flickinger JC. Cutaneous melanoma. In: De Vita Jr VT, Hellman S, Rosenberg AS, editors. Cancer: principles and practice of Oncology. Philadelphia: Lippincot Williams \& Wilkins; 2001. p.2012-2069.

3. Frija J, Bourrier P, Zagdanski AM, De Kerviler E. Diagnosis of a malignant lymph node. J Radiol. 2005;86:113-25.

4. Calvo López MJ, Vallejos Roca E, Muñoz Alcántara I, Navarro Díaz F, García Palacios MV. Ultrasonographic and power Doppler appearance of locoregional metastases from cutaneous melanoma. Radiologia. 2008;50:483-8.

5. Doubrovsky A, Scolyer RA, Murali R, McKenzie PR, Watson GF, Lee CS, et al. Diagnostic accuracy of fine needle biopsy for metastatic melanoma and its implications for patients management. Ann Surg Oncol. 2008;15:323-32.

6. van Rijk MC, Teertstra HJ, Peterse JL, Nieweg OE, Olmos RA, Hoefnagel CA, et al. Ultrasonography and fine-needle aspiration cytology in the preoperative evaluation of melanoma patients eligible for sentinel node biopsy. Ann Surg Oncol. 2006;13:1511-6.

7. Balch CM, Buzaid AC, Soong SJ, Atkins MB, Cascinelli N, Coit DG, et al. Final version of the American Joint Committee on Cancer Staging System for cutaneous melanoma. J Clin Oncol. 2001;19:3635-48.

8. Balch CM, Gershenwald JE, Soong SJ, Thompson JF, Atkins MB, Byrd DR, et al Final version of 2009 AJCC melanoma staging and classification. J Clin Oncol. 2009; 27:6199-206.

9. Lebsa-Weber A, Nunes DH, Souza Filho JJ, Carvalho-Pinto CJ. Avaliação de 496 laudos anatomopatológicos de melanoma diagnosticados no município de Florianópolis, Santa Catarina, Brasil. An Bras Dermatol. 2007;82:227-32.

10. Maia MA de OF, Funchal CSRZ, Ferrari Jr NM, Ribeiro MCS de A. Melanoma acrolentiginoso: um desafio ao diagnóstico precoce. An Bras Dermatol. 2003;78:553-60.

\footnotetext{
ENDEREÇO PARA CORRESPONDÊNCIA / MAILING ADDRESS:

Nurimar C. Fernandes

Rua Alexandre de Gusmão, 28 / 201

20520-120 Rio de Janeiro - RJ

Telefax: (21) 2568-4158

E-mail: nurimarfernandes@terra.com.br
}

Como citar este artigo/How to cite this article: Fernandes NC, Calmon R. Melanoma cutâneo: estudo prospectivo de 42 casos. An Bras Dermatol. 2010;86(6):1233-5. 